Bull. Austral. Math. Soc.

$49 \mathrm{~A} 40,54 \mathrm{H} 25,47 \mathrm{H} 10$

VOL. 41 (1990) [457-473]

\title{
A NEW MINIMAX INEQUALITY ON H-SPACES WITH APPLICATIONS
}

\author{
Xie Ping Ding, Won Kyu Kim and KoK-Keong Tan
}

\begin{abstract}
A new minimax inequality on $H$-spaces is obtained together with six equivalent formulations. As applications, some results on fixed point theorems and system of inequalities are proved. Our results generalise the corresponding results on (1) minimax inequalities due to Fan, Yen, Tan, Shih-Tan and Ding-Tan, (2) fixed point theorems due to Browder, Tarafdar, Shih-Tan and Ding-Tan, (3) convex inequalities due to Fan, (4) systems of inequalities due to Granas-Liu and (5) a minimax theorem due to Kneser.
\end{abstract}

\section{INTRODUCTION}

In 1961, Fan [13] gave an extension of the classical Knaster-KuratowskiMazurkiewicz theorem [20] to an arbitrary Hausdorff topological vector space. Since then this result has been widely used in non-linear functional analysis and is known as Fan's Lemma. Among various applications of Fan's Lemma, an application (both simple and non-trivial) is the celebrated 1972 minimax inequality of Fan [14]. The minimax inequality of Fan is of a topological nature and has important repercussions both in convex analysis and non-linear functional analysis.

Since 1972, there have been numerous generalisations of Ky Fan's minimax inequality by weakening the compactness assumption or the convexity assumption in topological vector spaces, for example see $[1,2,4,7,15,26,27,29,30]$. In $[17,18$, 19], Horvath obtained some minimax inequalities by replacing convexity with pseudoconvexity $[\mathbf{1 7}]$ or contractibility $[\mathbf{1 8}, \mathbf{1 9}]$ in topological spaces but only in a compact setting. In [3], using Horvath's approach in [19], Bardaro and Ceppitelli introduced the notions of " $H$-space", "weakly $H$-convex" and " $H$-compact" to obtain some minimax inequalities in non-compact setting for mappings taking values in an ordered vector space.

In this paper, we first give two versions of a new generalisation of the Ky Fan minimax inequality to non-compact $B$-spaces which include a generalisation of $\mathrm{Ky}$

Received 5 July 1989

This work was partially supported by NSERC of Canada under Grant A-8096, and for the second author by a grant from the Korea Science and Engineering Foundation in 1988 - 1989.

Copyright Clearance Centre, Inc. Serial-fee code: 0004-9729/90 \$A2.00+0.00. 
Fan's minimax inequality by Shih-Tan [26]. Next five equivalent formulations of our minimax inequality are given, extending some earlier results of Fan [13], Browder [5, 6], Shih-Tan [24, 26], Ding-Tan [10] and Tarafdar [28]. As applications, some results on system of inequalities are proved which generalise those of Granas-Liu [16] and Kneser $[21]$.

\section{Minimax inequalities}

Let $X$ be a non-empty set; we shall denote by $2^{X}$ the family of all non-empty subsets of $X$ and by $\mathcal{F}(X)$ the family of all non-empty finite subsets of $X$. If $F: X \rightarrow$ $2^{X}$, we define $F^{-1}, F^{*}, F^{c}$ by

$$
\begin{aligned}
F^{-1}(y) & =\{x \in X: y \in F(x)\}, F^{*}(y)=\{x \in X: y \notin F(x)\} \text { and } \\
F^{c}(x) & =\{y \in X: y \notin F(x)\} .
\end{aligned}
$$

The following notions, which were introduced by Bardaro and Ceppitelli in [3], were motivated by an earlier work of Horvath [19].

A pair $\left(X,\left\{F_{A}\right\}\right)$ is said to be an $H$-space if $X$ is a topological space (which need not be Hausdorff) and $\left\{F_{A}\right\}$ is a family of non-empty contractible subsets of $X$ indexed by $A \in \mathcal{F}(X)$ such that $F_{A} \subset F_{A^{\prime}}$ whenever $A \subset A^{\prime}$. Let $\left(X,\left\{F_{A}\right\}\right)$ be an $H$-space. A subset $D$ of $X$ is called $H$-convex if $F_{A} \subset D$ for each $A \in \mathcal{F}(D)$. A map $F: X \rightarrow 2^{X}$ is called $H-K K M$ if $F_{A} \subset \bigcup_{x \in A} F(x)$ for each $A \in \mathcal{F}(X)$.

The following lemma is a slight improvement of Corollary 1.1 of Horvath in [19].

Lemma 1. Let $\left(X,\left\{F_{A}\right\}\right)$ be an $H$-space and $G: X \rightarrow 2^{X}$ be such that

(a) $G$ is an $H-K K M$ map;

(b) for some $x_{0} \in X, G\left(x_{0}\right)$ is compact and for each $x \in X, G(x)$ is closed in $X$.

Then $\bigcap_{x \in X} G(x) \neq 0$.

Proof: From Theorem 1 of Horvath in [19], it follows that the family $\{G(x): x \in$ $X\}$ has the finite intersection property. By (b), $\bigcap_{x \in X} G(x) \neq \emptyset$.

TheOREM 1. Let $\left(X,\left\{F_{A}\right\}\right)$ be an $H$-space and $\phi, \psi: X \times X \rightarrow \mathbf{R} \cup\{-\infty, \infty\}$ be such that

(a) $\phi(x, y) \leqslant \psi(x, y)$ for each $(x, y) \in X \times X$ and $\psi(x, x) \leqslant 0$ for each $x \in X$;

(b) for each fixed $x \in X, \phi(x, y)$ is a lower semicontinuous function of $y$ on $X$;

(c) the map $x \rightarrow F(x)=\{y \in X: \psi(x, y) \leqslant 0\}$ is $H-K K M$; 
(d) there exist a non-empty closed and compact subset $K$ of $X$ and $x_{0} \in X$ such that $\psi\left(x_{0}, y\right)>0$ for all $y \in X \backslash K$.

Then there exists $\hat{y} \in X$ such that $\phi(x, \widehat{y}) \leqslant 0$ for all $x \in X$.

Proof: For each $x \in X$, let

$$
F(x)=\{y \in X: \psi(x, y) \leqslant 0\}, \quad G(x)=\{y \in X: \phi(x, y) \leqslant 0\}
$$

Then we have

(i) for each $x \in X, F(x) \subset G(x)$ by (a),

(ii) for each $x \in X, G(x)$ is closed in $X$ by (b),

(iii) by (a) and (d), $F\left(x_{0}\right) \subset K$ so that $\overline{F\left(x_{0}\right)} \subset \bar{K}=K$ and hence $\overline{F\left(x_{0}\right)}$ is compact.

By Lemma 1, $\bigcap_{x \in X} \overline{F(x)} \neq \emptyset$. Hence, it follows from (i) and (ii) that $\bigcap_{x \in X} G(x) \neq \emptyset$. Take any $\widehat{y} \in \bigcap_{x \in X} G(x) ;$ then $\phi(x, \widehat{y}) \leqslant 0$ for all $x \in X$.

Lemma 2. Let $\left(X,\left\{F_{A}\right\}\right)$ be an $H$-space and $\psi: X \times X \rightarrow \mathbf{R}$ be such that $\psi(x, x) \leqslant 0$ for all $x \in X$.

Then the following two conditions are equivalent:

(1) the map $x \rightarrow F(x)=\{y \in X: \psi(x, y) \leqslant 0\}$ is $H-K K M$;

(2) for each $A \in \mathcal{F}(X)$ and for each $y \in F_{A}, \min _{x \in A} \psi(x, y) \leqslant 0$.

Proof: $(1) \Rightarrow(2)$ : Suppose (2) does not hold; then there exist $A \in \mathcal{F}(X)$ and $y \in F_{A}$ such that $\min _{x \in A} \psi(x, y)>0$; it follows that $y \notin f(x)$ for all $x \in A$ so that $y \notin \bigcup_{x \in A} F(x)$. This contradicts (1) that $F_{A} \subset \bigcup_{x \in A} F(x)$ and $y \in F_{A}$.

$(2) \Rightarrow(1)$ : Suppose (1) does not hold; then there exists $A \in \mathcal{F}(X)$ such that $F_{A} \not \subset \bigcup_{x \in A} F(x)$. Choose any $y \in F_{A}$ such that $y \notin \bigcup_{x \in A} F(x)$; it follows that $\psi(x, y)>0$ for all $x \in A$ so that $\min _{x \in A} \psi(x, y)>0$ which contradicts (2).

The condition (2) in Lemma 2 generalises the notion of 0 -diagonally quasi-concave in $y$ introduced in [31]. In view of Lemma 2, we have the following result which is equivalent to Theorem 1.

THEOREM 2. Let $\left(X,\left\{F_{A}\right\}\right)$ be an $H$-space and $\phi, \psi: X \times X \rightarrow \mathrm{R} \cup\{-\infty, \infty\}$ be such that

(a) $\phi(x, y) \leqslant \psi(x, y)$ for each $(x, y) \in X \times X$ and $\psi(x, x) \leqslant 0$ for each $x \in X$;

(b) for each fixed $x \in X, \phi(x, y)$ is a lower semicontinuous function of $y$ on $\boldsymbol{X}$; 
(c) for each $A \in \mathcal{F}(X)$ and for each $y \in F_{A}, \min _{x \in A} \psi(x, y) \leqslant 0$;

(d) there exist a non-empty closed and compact subset $K$ of $X$ and $x_{0} \in X$ such that $\psi\left(x_{0}, y\right)>0$ for all $y \in X \backslash K$.

Then there exists $\widehat{y} \in X$ such that $\phi(x, \widehat{y}) \leqslant 0$ for all $x \in X$.

Theorem 2 generalises Theorem 1 of Shih-Tan [26] to $H$-spaces.

Corollary 1. Let $X$ be a non-empty convex subset of a topological vector space and $\phi, \psi: X \times X \rightarrow \mathbf{R} \cup\{-\infty, \infty\}$ be such that

(a) $\phi(x, y) \leqslant \psi(x, y)$ for each $(x, y) \in X \times X$ and $\psi(x, x) \leqslant 0$ for each $x \in X$;

(b) for each fixed $x \in X, \phi(x, y)$ is a lower semicontinuous function of $y$ on $X$;

(c) for each $A \in \mathcal{F}(X)$ and for each $y \in \operatorname{co}(A), \min _{x \in A} \psi(x, y) \leqslant 0$;

(d) there exist a non-empty closed and compact subset $K$ of $X$ and $x_{0} \in X$ such that $\psi\left(x_{0}, y\right)>0$ for all $y \in X \backslash K$.

Then there exists $\widehat{y} \in X$ such that $\phi(x, \widehat{y}) \leqslant 0$ for all $x \in X$.

Proof: For each $A \in \mathcal{F}(X)$, let $F_{A}=\operatorname{co}(A)$; then $\left(X,\left\{F_{A}\right\}\right)$ forms an $H$-space so that the conclusion follows from Theorem 2 .

We emphasise here that, in Theorem 1 (also Theorem 2), the coercive condition (d) is imposed on $\psi$ while in other non-compact generalisations of Fan's minimax inequality, the coercive condition is imposed on $\phi$, for example see Allen [1], Tan [27], Bae-KimTan [2] and Ding-Tan [7]. For the purpose of comparision, we shall state the minimax inequality of Ding-Tan [7, Theorem 2] as follows:

TheOREM A. Let $X$ be a non-empty convex set in a topological vector space and $\phi, \psi: X \times X \rightarrow R \cup\{-\infty, \infty\}$ be such that

(a) $\phi(x, y) \leqslant \psi(x, y)$ for all $(x, y) \in X \times X$ and $\psi(x, x) \leqslant 0$ for all $x \in X$;

(b) for each fixed $x \in X, \phi(x, y)$ is a lower semicontinuous function of $y$ on $C$ for each non-empty compact subset $C$. of $X$;

(c) for each $y \in X$, the set $\{x \in X: \psi(x, y)>0\}$ is convex;

(d) there exist a non-empty compact convex subset $X_{0}$ of $X$ and a nonempty compact subset $K$ of $X$ such that for each $y \in X \backslash K$, there exists $x \in \operatorname{co}\left(X_{0} \cup\{y\}\right)$ with $\phi(x, y)>0$.

Then there exists $\widehat{y} \in K$ such that $\phi(x, \widehat{y}) \leqslant 0$ for all $x \in X$.

For generalisations of Theorem A to $H$-spaces, we refer to Ding-Tan $[8,9]$.

The following examples show that the coercive conditions (d) of Theorem 1 and (d) of Theorem A are independent of each other: 
EXAmple 1. Let $X=[0, \infty)$ and $\phi, \psi: X \times X \rightarrow \mathbf{R}$ be defined by

$$
\begin{aligned}
& \phi(x, y)= \begin{cases}1 & \text { if }(x, y) \in\left\{(x, y) \in \mathbf{R} \times \mathbf{R}: x<y<x+\frac{1}{1+x} \text { for } 0 \leqslant x<1\right\} \\
0 & \text { otherwise }\end{cases} \\
& \psi(x, y)= \begin{cases}0 & \text { if } y \leqslant x \\
1 & \text { otherwise. }\end{cases}
\end{aligned}
$$

Then all the conditions (a), (b), (c) and (d) in Theorem 1 are satisfied with $K=\{0\}=$ $\left\{x_{0}\right\}$ so that there exists $\widehat{y}=0 \in X$ such that $\phi(x, \widehat{y}) \leqslant 0$ for all $x \in X:$ However, there does not exist a non-empty compact convex subset $X_{0}$ of $X$ and a non-empty compact subset $K$ of $X$ such that for each $y \in X \backslash K$, there exists a point $x \in \operatorname{co}\left(X_{0} \cup\{y\}\right)$ with $\phi(x, y)>0$. Thus Theorem $\mathrm{A}$ is not applicable.

EXAMPLE 2. Let $X=[0, \infty)$ and $\phi, \psi: X \times X \rightarrow \mathbf{R}$ be defined by

$$
\phi(x, y)=\psi(x, y)= \begin{cases}0 & \text { if } y \leqslant x \text { or } x+\frac{1}{1+x} \leqslant y, \\ 1 & \text { otherwise. }\end{cases}
$$

Then all the conditions (a), (b), (c) and (d) in Theorem $A$ are satisfied with $X_{0}=K=\{0\}$ so that there exists $\hat{y}=0 \in X$ such that $\phi(x, \widehat{y}) \leqslant 0$ for all $x \in X$. However, there does not exist a non-empty compact subset $K$ of $X$ and $x_{0} \in X$ such that $\psi\left(x_{0}, y\right)>0$ for all $y \in X \backslash K$. Therefore Theorem 1 is not applicable.

\section{EQUIVALENT FORMULATIONS OF.THE MINIMAX INEQUALITY}

The following Theorems $3,4,5,6$ and 7 are all equivalent to our minimax inequality Theorem 2 (and hence also Theorem 1):

Theorem 3. (First Geometric Form) Let $\left(X,\left\{F_{A}\right\}\right)$ be an $H$-space and $B$, $C \subset X \times X$ be such that

(a) $B \subset C$;

(b) for each $x \in X$, the set $\{y \in X:(x, y) \in B\}$ is open in $X$;

(c) for each $A \in \mathcal{F}(X)$ and for each $y \in F_{A}$, there exists $x \in A$ such that $(x, y) \notin C$;

(d) there exist a non-empty closed and compact subset $K$ of $X$ and $x_{0} \in X$ such that $\left(x_{0}, y\right) \in C$ for all $y \in X \backslash K$.

Then either there exists $\widehat{x} \in X$ such that $(\widehat{x}, \widehat{x}) \in C$ or there exists $\widehat{y} \in X$ such that $\{x \in X:(x, \widehat{y}) \in B\}=\emptyset$.

Theorem 4. (Second Geometric Form) Let $\left(X,\left\{F_{A}\right\}\right)$ be an $H$-space and $M$, $N \subset X \times X$ be such that

(a) $N \subset M$; 
(b) for each $x \in X$, the set $\{y \in X:(x, y) \in M\}$ is closed in $X$;

(c) for each $A \in \mathcal{F}(X)$ and for each $y \in F_{A}$, there exists $x \in A$ such that $(x, y) \in N$;

(d) there exist a non-empty closed and compact subset $K$ of $X$ and $x_{0} \in X$ such that $\left(x_{0}, y\right) \notin N$ for all $y \in X \backslash K$.

Then either there exists $\widehat{x} \in X$ such that $(\widehat{x}, \widehat{x}) \notin N$ or there exists $\widehat{y} \in X$ such that $X \times\{\hat{y}\} \subset M$.

Theorem 5. (Fixed Point Version) Let $\left(X,\left\{F_{A}\right\}\right)$ be an $H$-space and $F, G$ : $X \rightarrow 2^{X} \cup\{0\}$ be such that

(a) for each $x \in X, F(x) \subset G(x)$;

(b) for each $x \in X, F(x)$ is open in $X$;

(c) for each $A \in \mathcal{F}(X)$ and for each $y \in F_{A}$, there exists $x \in A$ such that $y \notin G(x)$;

(d) there exist a non-empty closed and compact subset $K$ of $X$ and $x_{0} \in X$ such that $X \backslash K \subset G\left(x_{0}\right)$;

(e) for each $y \in X, F^{-1}(y) \neq \emptyset$.

Then there exists $\widehat{x} \in X$ such that $\widehat{x} \in G(\widehat{x})$.

Theorem 6. (Fixed Point Version) Let $\left(X,\left\{F_{A}\right\}\right)$ be an $H$-space and $S$, $T: X \rightarrow 2^{X}$ be such that

(a) for each $x \in X, S(x) \subset T(x)$;

(b) for each $y \in X, S^{-1}(y)$ is open in $X$;

(c) for each $A \in \mathcal{F}(X)$ and for each $y \in F_{A}$, there exists $x \in A$ such that $x \notin T(y)$;

(d) there exist a non-empty closed and compact subset $K$ of $X$ and $x_{0} \in X$ such that $X \backslash K \subset T^{-1}\left(x_{0}\right)$.

Then there exists $\widehat{x} \in X$ such that $\widehat{x} \in T(\widehat{x})$.

Theorem 7. (Maximal Element Version) Let $\left(X,\left\{F_{A}\right\}\right)$ be an $H$-space and $P$, $Q: X \rightarrow 2^{X} \cup\{\emptyset\}$ be such that

(a) for each $x \in X, P(x) \subset Q(x)$ and $x \notin Q(x)$;

(b) for each $y \in X, P^{-1}(y)$ is open in $X$;

(c) for each $A \in \mathcal{F}(X)$ and for each $y \in F_{A}$, there exists $x \in A$ such that $x \notin Q(y)$;

(d) there exist a non-empty closed and compact subset $K$ of $X$ and $x_{0} \in X$ such that $X \backslash K \subset Q^{-1}\left(x_{0}\right)$.

Then there exists $\widehat{x} \in X$ such that $P(\widehat{x})=\emptyset$.

Proof of “Theorem $2 \Rightarrow$ TheOREM $3^{\text {" : }}$ 
Suppose that for each $x \in X,(x, x) \notin C$. Let $\phi$ and $\psi$ be the characteristic functions of $B$ and $C$ respectively, then

(i) $\phi(x, y) \leqslant \psi(x, y)$ for each $(x, y) \in X \times X$ by (a) and $\psi(x, x) \leqslant 0$ by assumption;

(ii) for each $x \in X$ and for each $\lambda \in \mathbf{R}$,

$$
\{y \in X: \phi(x, y)>\lambda\}= \begin{cases}X, & \text { if } \lambda<0, \\ \{y \in X:(x, y) \in B\}, & \text { if } 0 \leqslant \lambda<1, \\ \emptyset, & \text { if } \lambda \geqslant 1,\end{cases}
$$

is open in $X$ by (b) so that $\phi(x, y)$ is a lower semicontinuous function of $\boldsymbol{y}$ on $\boldsymbol{X}$;

(iii) by (c) and (d), the conditions (c) and (d) of Theorem 2 hold.

By Theorem 2 , there exists $\hat{y} \in X$ such that $\phi(x, \widehat{y}) \leqslant 0$ for all $x \in X$. It follows that $(x, \widehat{y}) \notin B$ for all $x \in X$; that is, $\{x \in X:(x, \widehat{y}) \in B\}=\emptyset$.

Proof of "ThEOREM $3 \Rightarrow$ ThEOREM 2 ":

Let $B, C \subset X \times X$ be defined by

$$
\begin{aligned}
& B=\{(x, y) \in X \times X: \phi(x, y)>0\} \\
& C=\{(x, y) \in X \times X: \psi(x, y)>0\}
\end{aligned}
$$

Then the conditions (a), (b), (c) and (d) of Theorem 3 are all satisfied and $(x, x) \notin C$ for all $x \in X$. Hence by Theorem 3, there exists $\widehat{y} \in X$ such that $\{x \in X:(x, \widehat{y}) \in B\}=\emptyset$; that is $\phi(x, \widehat{y}) \leqslant 0$ for all $x \in X$.

PROOF OF "ThEOREM $3 \Rightarrow$ ThEOREM 4 ":

Let $B=X \times X \backslash M$ and $C=X \times X \backslash N$; apply Theorem 3, the conclusion follows.

Proof of "ThEOREM $4 \Rightarrow$ ThEOREM 3":

Let $M=X \times X \backslash B$ and $N=X \times X \backslash C$; apply Theorem 4, the conclusion follows.

Proof of "Theorem $3 \Rightarrow$ Theorem 5":

Let $B, C \subset X \times X$ be defined by

$$
\begin{aligned}
& B=\{(x, y) \in X \times X: y \in F(x)\} \\
& C=\{(x, y) \in X \times X: y \in G(x)\} .
\end{aligned}
$$

Then the conditions (a), (b), (c) and (d) in Theorem 3 are all satisfied and by (e), for each $y \in X$, there exists $x \in X$ such that $(x, y) \in B$ so that the set $\{x \in X$ : 
$(x, y) \in B\} \neq \emptyset$. Hence by Theorem 3, there exists $\widehat{x} \in X$ such that $(\widehat{x}, \widehat{x}) \in C$; that is, $\widehat{x} \in G(\widehat{x})$.

Proof of "Theorem $5 \Rightarrow$ Theorem 3 ":

Define $F, G: X \rightarrow 2^{X} \cup\{\theta\}$ by

$$
\begin{aligned}
& F(x)=\{y \in X:(x, y) \in B\}, \\
& G(x)=\{y \in X:(x, y) \in C\}
\end{aligned}
$$

for each $x \in X$. Then the conditions (a), (b), (c) and (d) in Theorem 5 are all satisfied. Hence by Theorem 5, either there exists $\widehat{y} \in X$ such that $F^{-1}(\widehat{y})=\emptyset$ or there exists $\widehat{x} \in X$ such that $\widehat{x} \in G(\widehat{x})$; that is, either there exists $\widehat{y} \in X$ such that $\{x \in X:(x, \widehat{y}) \in B\}=\emptyset$ or there exists $\widehat{x} \in X$ such that $(\widehat{x}, \widehat{x}) \in C \ldots$

PROOF OF "ThEOREM $5 \Rightarrow$ THEOREM 6 ":

Define $F, G: X \rightarrow 2^{X} \cup\{\emptyset\}$ by $F=S^{-1}$ and $G=T^{-1}$; then by (a), (b), (c) and (d), the conditions (a), (b), (c) and (d) of Theorem 5 are all satisfied. Since $S(x) \neq \emptyset$ for all $x \in X$, the condition (e) of Theorem 5 is also satisfied. Hence by Theorem 5, there exists $\widehat{x} \in X$ such that $\widehat{x} \in G(\widehat{x})$; that is, $\widehat{x} \in T(\widehat{x})$.

Proof of "Theorem $6 \Rightarrow$ TheOREM 5 ":

Define $S, T: X \rightarrow 2^{X} \cup\{0\}$ by $S=F^{-1}$ and $T=G^{-1}$; then by (a), (b), (c) (d), the conditions (a), (b), (c) and (d) of Theorem 6 are all satisfied. By (e), $S(x) \neq \emptyset$ for each $x \in X$ so that $S, T: X \rightarrow 2^{X}$. By Theorem 6 , there exists $\widehat{x} \in X$ such that $\widehat{x} \in T(\widehat{x})$; that is, $\widehat{x} \in G(\widehat{x})$.

The proof of "Theorem $6 \Leftrightarrow$ Theorem 7" is obvious.

As an immediate consequence of Theorem 3 , we have the following result which generalises Theorem 3 of Shih-Tan [24] in several aspects:

Corollary 2. Let $X$ be a non-empty convex subset of a topological vector space and $B, C \subset X \times X$ be such that

(a) $B \subset C$;

(b) for each $x \in X$, the set $\{y \in X:(x, y) \in B\}$ is open in $X$;

(c) for each $A \in \mathcal{F}(X)$ and for each $y \in \operatorname{co}(A)$, there exists $x \in A$ such that $(x, y) \notin C ;$

(d) there exist a non-empty closed and compact subset $K$ of $X$ and $x_{0} \in X$ such that $\left(x_{0}, y\right) \in C$ for all $y \in X \backslash K$.

Then either there exists $\widehat{x} \in X$ such that $(\widehat{x}, \widehat{x}) \in C$ or there exists $\widehat{y} \in X$ such that $\{x \in X:(x, \widehat{y}) \in B\}=\emptyset$.

Similarly, Theorem 4 generalises Theorem 4 of Shih-Tan [24] to $H$-spaces in noncompact setting. 
Corollary 3. Let $\left(X,\left\{F_{A}\right\}\right)$ be an $H$-space and $F, G: X \rightarrow 2^{X} \cup\{\emptyset\}$ be such that

(a) for each $x \in X, F(x) \subset G(x)$;

(b) for each $x \in X, F(x)$ is open in $X$;

(c) for each $y \in X, F^{-1}(y) \neq \emptyset$ and $G^{-1}(y)$ is $H$-convex;

(d) there exist a non-empty closed and compact subset $K$ of $X$ and $x_{0} \in X$ such that $X \backslash K \subset G\left(x_{0}\right)$.

Then there exists $\widehat{x} \in X$ such that $\widehat{x} \in G(\widehat{x})$.

Proof: Suppose there exist $A \in \mathcal{F}(X)$ and $y \in F_{A}$ such that $y \in G(x)$ for all $x \in A$, then $A \subset G^{-1}(y)$ so that by (c), $F_{A} \subset G^{-1}(y)$ and hence $y \in F_{A} \subset G^{-1}(y)$; thus $y \in G(y)$. Suppose for each $A \in \mathcal{F}(X)$ and for each $y \in F_{A}$, there exists $x \in A$ such that $y \notin G(x)$, then the condition (c) of Theorem 5 is also satisfied. By Theorem 5 , there exists $\widehat{x} \in X$ such that $\widehat{x} \in G(\widehat{x})$.

Corollary 3 generalises Theorem 5 of Shih-Tan [24] and its non-compact generalisation by Shih-Tan [26, Theorem 2] to non-comvex setting.

We remark here that Theorem 7 , the maximal element version of minimax inequality, has applications to mathematical economics, for example see Ding-Tan [10].

The following result is an easy consequence of Theorem 6:

Corollary 4. Let $\left(X,\left\{F_{A}\right\}\right)$ be an $H$-space and $S, T: X \rightarrow 2^{X}$ be such that

(a) for each $x \in X, S(x) \subset T(x)$;

(b) for each $y \in X, S^{-1}(y)$ is open in $X$;

(c) for each $x \in X, T(x)$ is $H$-convex;

(d) there exist a non-empty closed and compact subset $K$ of $X$ and $x_{0} \in X$ such that $X \backslash K \subset T^{-1}\left(x_{0}\right)$.

Then there exists $\widehat{x} \in X$ such that $\widehat{x} \in T(\widehat{x})$.

Proof: Suppose there exist $A \in \mathcal{F}(X)$ and $y \in F_{A}$ such that $x \in T(y)$ for all $x \in A$, then $A \subset T(y)$ so that by (c), $F_{A} \subset T(y)$ and hence $y \in T(y)$ as $y \in F_{A}$. If for each $A \in \mathcal{F}(X)$ and for each $y \in F_{A}$, there exists $x \in A$ such that $x \notin T(y)$, then the condition (c) of Theorem 6 is also satisfied. By Theorem 6 , there exists $\widehat{x} \in X$ such that $\widehat{x} \in T(\widehat{x})$.

Corollary 4 generalises Theorem 1 of Browder in [5] (which is equivalent to Lemma 4 of Fan in [13]) and its non-compact generalisation by Browder [6, Theorem 2] in several aspects. Clearly Corollary 4 is equivalent to Corollary 3 . We shall now give another equivalent formulation of Corollary 4 as follows:

Corollary 5. Let $\left(X,\left\{\mathcal{F}_{A}\right\}\right)$ be an $B$-space and $T: X \rightarrow 2^{X} \cup\{0\}$ be such 
that

(i) for each $x \in X, T(x)$ is $H$-convex;

(ii) for each $y \in X, T^{-1}(y)$ contains an open subset $O_{y}$ (which may be empty) of $X$ such that $\bigcup_{y \in X} O_{y}=X$;

(iii) there exist a non-empty closed and compact subset $K$ of $X$ and $x_{0} \in X$ such that $X \backslash K \subset T^{-1}\left(x_{0}\right)$.

Then there exists $\widehat{x} \in X$ such that $\widehat{x} \in T(\widehat{x})$.

Proof of "Corollary $4 \Rightarrow$ Corollary 5":

Define $S: X \rightarrow 2^{X}$ by $S(x)=\left\{y \in X: x \in O_{y}\right\}$ for each $x \in X$. Then we have

(1) for each $x \in X, S(x) \neq \emptyset$ and $S(x) \subset T(x)$ by (ii);

(2) for each $y \in X, S^{-1}(y)=O_{y}$ is open in $X$ by (ii).

Thus all the hypotheses of Corollary 4 are satisfied and hence the conclusion follows from Corollary 4.

Proof of "Corollary $5 \Rightarrow$ Corollary 4":

For each $y \in X$, let $O_{y}=S^{-1}(y)$; then

(1) for each $y \in X, O_{y}$ is open in $X$ by (b) and $O_{y}=S^{-1}(y) \subset T^{-1}(y)$ by (a);

(2) by the assumption, for each $x \in X, S(x) \neq \emptyset$, we have $\bigcup_{y \in X} O_{y}=$ $\bigcup_{y \in X} S^{-1}(y)=X$

By Corollary 5, the conclusion of Corollary 4 holds.

Corollary 5 generalises Theorem 2 of Ding-Tan [10] which in turn generalises Theorem 1 of Tarafdar in [28] (also Theorem 1 of Mehta-Tarafdar in [22]) to non-compact $H$-spaces.

\section{Systems of inEqualities}

According to Pietsch [23, p.40], a collection $\mathcal{F}$ of real-valued functions $f$ defined on a set $X$ is called concave if, given any finite subset $\left\{f_{1}, \ldots, f_{n}\right\}$ of $\mathcal{F}$ and $\alpha_{1}, \ldots, \alpha_{n} \geqslant 0$ with $\sum_{i=1}^{n} \alpha_{i}=1$, there exists $f \in \mathcal{F}$ such that $f(x) \geqslant \sum_{i=1}^{n} \alpha_{i} f_{i}(x)$ for all $x \in X$.

Given any two collections $\mathcal{F}$ and $\mathcal{G}$ of real-valued functions on a set $X$, we shall write $\mathcal{F} \leqslant \mathcal{G}$ if for any $f \in \mathcal{F}$, there exists $g \in \mathcal{G}$ such that $f(x) \leqslant g(x)$ for all $x \in X$.

ThEOREM 8. Let $\left(X,\left\{F_{A}\right\}\right)$ be a normal $H$-space. Let $\mathcal{F}, \mathcal{G}$ and $\mathcal{H}$ be three collections of real-valued functions on $X$ such that

(i) $\mathcal{F} \leqslant \mathcal{G} \leqslant \mathcal{H}$; 
(ii) for each $f \in \mathcal{F}, f$ is lower semicontinuous on $X$;

(iii) for any finite subset $\left\{g_{1}, \ldots, g_{n}\right\}$ of $\mathcal{G}$ and for any $\beta_{1}, \ldots, \beta_{n}: X \rightarrow[0,1]$ with $\sum_{i=1}^{n} \beta_{i}(x)=1$ for each $x \in X$, the following property holds: for each $A \in \mathcal{F}(X)$ and for each $y \in F_{A}$, there exists $x \in A$ such that

$$
\sum_{i=1}^{n} \beta_{i}(y) g_{i}(y) \leqslant \sum_{i=1}^{n} \beta_{i}(y) g_{i}(x)
$$

(iv) there exist a non-empty closed and compact subset $K$ of $X$ and $x_{0} \in X$ such that for any finite subset $\left\{g_{1}, \ldots, g_{n}\right\}$ of $\mathcal{G}$ and for any $\beta_{1}, \ldots, \beta_{n}: X \rightarrow[0,1]$ with $\sum_{i=1}^{n} \beta_{i}(x)=1$ for each $x \in X$,

$$
\sum_{i=1}^{n} \beta_{i}(y) g_{i}(y)>\sum_{i=1}^{n} \beta_{i}(y) g_{i}\left(x_{0}\right) \text { for all } y \in X \backslash K
$$

(v) the collection $\mathcal{H}$ is concave.

Then given any $\rho \in \mathbf{R}$, one of the following properties holds:

(1) there exists an $h \in \mathcal{H}$ such that $\inf _{x \in X} h(x)>\rho$;

(2) there exists a point $\hat{y} \in X$ such that $f(\widehat{y}) \leqslant \rho$ for all $f \in \mathcal{F}$.

Proof: Without loss of generality, we may assume that $\rho=0$. For each $f \in \mathcal{F}$, let $Q(f)=\{x \in K: f(x) \leqslant 0\}$; then $Q(f)$ is closed in $K$ by (ii). If the family $\{Q(f): f \in \mathcal{F}\}$ has the finite intersection property, then by the compactness of $K$ we obtain the alternative (2). Suppose $\{Q(f): f \in \mathcal{F}\}$ does not have the finite intersection property. Then there are $f_{1}, \ldots, f_{n} \in \mathcal{F}$ such that $\bigcap_{i=1}^{n} Q\left(f_{i}\right)=\emptyset$. For each $i=1, \ldots, n$, let $V_{i}=X \backslash Q\left(f_{i}\right)$; then each $V_{i}$ is open in $X$ and $\left\{V_{1}, \ldots, V_{n}\right\}$ is an open covering of the normal space $X$. Let $\left\{\beta_{1}, \ldots, \beta_{n}\right\}$ be a continuous partition of unity, subordinate to this open covering. Thus for each $i=1, \ldots, n, \beta_{i}: X \rightarrow[0,1]$ is continuous and $\operatorname{Supp} \beta_{i} \subset V_{i}$ such that $\sum_{i=1}^{n} \beta_{i}(x)=1$ for each $x \in X$. Choose $g_{1}, \ldots, g_{n} \in \mathcal{G}$ and $h_{1}, \ldots, h_{n} \in \mathcal{H}$ such that $f_{i} \leqslant g_{i} \leqslant h_{i}$ on $X$ for each $i=1, \ldots, n$. Define $\phi, \psi: X \times X \rightarrow \mathbf{R}$ as follows:

$$
\begin{aligned}
& \phi(x, y)=\sum_{i=1}^{n} \beta_{i}(y) f_{i}(y)-\sum_{i=1}^{n} \beta_{i}(y) g_{i}(x), \\
& \psi(x, y)=\sum_{i=1}^{n} \beta_{i}(y) g_{i}(y)-\sum_{i=1}^{n} \beta_{i}(y) g_{i}(x) .
\end{aligned}
$$


Then we have

(1) $\phi(x, y) \leqslant \psi(x, y)$ for each $(x, y) \in X \times X$ and $\psi(x, x)=0$ for all $x \in X$;

(2) for each fixed $x \in X, \phi(x, y)$ is a lower semicontinuous function of $y$ on $\boldsymbol{X}$ by (ii);

(3) by (iii), for each $A \in \mathcal{F}(X)$ and for each $y \in F_{A}$, there exists $x \in A$ such that $\psi(x, y) \leqslant 0$; thus $\min _{x \in A} \psi(x, y) \leqslant 0$;

(4) by (iv), there exist a non-empty closed and compact subset $K$ of $X$ and $x_{0} \in X$ such that $\psi\left(x_{0}, y\right)>0$ for all $y \in X \backslash K$.

Thus all hy potheses of Theorem 2 are satisfied so that there exist $\widehat{y} \in X$ such that $\phi(x, \widehat{y}) \leqslant 0$ for all $x \in X$; that is,

$$
\sum_{i=1}^{n} \beta_{i}(\widehat{y}) f_{i}(\widehat{y}) \leqslant \sum_{i=1}^{n} \beta_{i}(\widehat{y}) g_{i}(x) \text { for all } x \in X .
$$

By $(v)$, there is an $h \in \mathcal{H}$ satisfying $h(x) \geqslant \sum_{i=1}^{n} \beta_{i}(\widehat{y}) h_{i}(x)$ for all $x \in X$. Therefore for all $x \in X$,

$$
0<\sum_{i=1}^{n} \beta_{i}(\widehat{y}) f_{i}(\widehat{y}) \leqslant \sum_{i=1}^{n} \beta_{i}(\widehat{y}) g_{i}(x) \leqslant \sum_{i=1}^{n} \beta_{i}(\widehat{y}) h_{i}(x) \leqslant h(x) .
$$

This proves the alternative (1)

Theorem 8 generalises Fan's Lemma [23, p.40] (see also Theorem 6 in [25] which is equivalent to Theorem 1 of $\operatorname{Fan}$ in [12]) to $H$-spaces.

Let $h$ be a real-valued function defined on the product set $X \times Y$ of two arbitrary non-empty sets $X$ and $Y$. According to Fan [11], $h$ is said to be concave on $X$ if for any two elements $x_{1}, x_{2} \in X$ and two numbers $\alpha_{1}, \alpha_{2} \geqslant 0$ with $\alpha_{1}+\alpha_{2}=1$, there exists $x_{0} \in X$ such that

$$
h\left(x_{0}, y\right) \geqslant \alpha_{1} h\left(x_{1}, y\right)+\alpha_{2} h\left(x_{2}, y\right) \text { for all } y \in Y .
$$

TheOREM 9. Let $X$ be an arbitrary non-empty set and $\left(Y,\left\{F_{A}\right\}\right)$ be a normal $H$-space. Let $f, g, h: X \times Y \rightarrow \mathrm{R}$ be such that

(i) $f \leqslant g \leqslant h$ on $X \times Y$;

(ii) for each fixed $x \in X, f(x, y)$ is a lower semicontinuous function of $y$ on $Y$;

(iii) for any finite subset $\left\{x_{1}, \ldots, x_{n}\right\}$ of $X$ and for any $\beta_{1}, \ldots, \beta_{n}: Y \rightarrow[0,1]$ with $\sum_{i=1}^{n} \beta_{i}(y)=1$ for each $y \in Y$, the following property holds: for each 
$A \in \mathcal{F}(Y)$ and for each $y \in F_{A}$, there exists $z \in A$ such that

$$
\sum_{i=1}^{n} \beta_{i}(y) g\left(x_{i}, y\right) \leqslant \sum_{i=1}^{n} \beta_{i}(y) g\left(x_{i}, z\right)
$$

(iv) there exist a non-empty closed and compact subset $K$ of $Y$ and $z_{0} \in Y$ such that for any finite subset $\left\{x_{1}, \ldots, x_{n}\right\}$ of $X$ and for any $\beta_{1}, \ldots, \beta_{n}: Y \rightarrow[0,1]$ with $\sum_{i=1}^{n} \beta_{i}(y)=1$ for each $y \in Y$,

$$
\sum_{i=1}^{n} \beta_{i}(y) g\left(x_{i}, y\right)>\sum_{i=1}^{n} \beta_{i}(y) g\left(x_{i}, z_{0}\right) \text { for all } y \in Y \backslash K ;
$$

(v) $h$ is concave on $X$.

Then there exists $\widehat{y} \in Y$ such that

$$
\sup _{x \in X} f(x, \widehat{y}) \leqslant \sup _{x \in X} \inf _{\boldsymbol{y} \in Y} h(x, y) .
$$

Proof: Let $\rho=\sup _{x \in X} \inf _{y \in Y} h(x, y)$. Applying Theorem 8 with $X$ being the index set, there exists $\widehat{y} \in Y$ such that $f(x, \widehat{y}) \leqslant \rho$ for all $x \in X$. Hence the conclusion $f(x, \widehat{y}) \leqslant \rho$ follows.

Theorem 9 generalises the corresponding result due to Granas-Liu [16] to nonconvex and non-compact setting.

When $f=g=h$ in Theorem 9, we obtain the following new minimax theorem:

THEOREM 10. Let $X$ be an arbitrary non-empty set and $\left(Y,\left\{F_{A}\right\}\right)$ be a normal $H$-space. Let $g: X \times Y \rightarrow R$ be such that

(i) for each fixed $x \in X, g(x, y)$ is a lower semicontinuous function of $y$ on $Y$;

(ii) for any finite subset $\left\{x_{1}, \ldots, x_{n}\right\}$ of $X$ and for any $\beta_{1}, \ldots, \beta_{n}: Y \rightarrow[0,1]$ with $\sum_{i=1}^{n} \beta_{i}(y)=1$ for each $y \in Y$, the following property holds: for each $A \in \mathcal{F}(Y)$ and for each $y \in F_{A}$, there exists $z \in A$ such that

$$
\sum_{i=1}^{n} \beta_{i}(y) g\left(x_{i}, y\right) \leqslant \sum_{i=1}^{n} \beta_{i}(y) g\left(x_{i}, z\right)
$$

(iii) there exist a non-empty closed and compact subset $K$ of $Y$ and $z_{0} \epsilon$ $Y$ such that, for any finite subset $\left\{x_{1}, \ldots, x_{n}\right\}$ of $X$ and for any $\beta_{1}, \ldots, \beta_{n}: Y \rightarrow[0,1]$ with $\sum_{i=1}^{n} \beta_{i}(y)=1$ for each $y \in Y$,

$$
\sum_{i=1}^{n} \beta_{i}(y) g\left(x_{i}, y\right)>\sum_{i=1}^{n} \beta_{i}(y) g\left(x_{i}, z_{0}\right) \text { for all } y \in Y \backslash K
$$

(iv) $g$ is concave on $X$. 
Then

$$
\inf _{y \in Y} \sup _{x \in X} g(x, y)=\sup _{x \in X} \inf _{y \in Y} g(x, y) \text {. }
$$

Proof: By Theorem 9, we have $\inf _{y \in Y} \sup _{x \in X} g(x, y) \leqslant \sup _{x \in X} \inf _{y \in Y} g(x, y)$. The reverse inequality always holds.

In Theorem 10, if $Y$ is also compact, by taking $K=Y$ the condition (iii) is satisfied trivially. Hence we have the following immediate consequence of Theorem 10:

THEOREM 11. Let $X$ be an arbitrary non-empty set and $\left(Y,\left\{F_{A}\right\}\right)$ be a compact $H$-space. Let $g: X \times Y \rightarrow \mathrm{R}$ be such that

(i) for each fixed $x \in X, g(x, y)$ is a lower semicontinuous function of $y$ on $Y$;

(ii) for any finite subset $\left\{x_{1}, \ldots, x_{n}\right\}$ of $X$ and for any $\beta_{1}, \ldots, \beta_{n}: Y \rightarrow[0,1\}$ with $\sum_{i=1}^{n} \beta_{i}(y)=1$ for each $y \in Y$, the following property holds: for each $A \in \mathcal{F}(Y)$ and for each $y \in F_{A}$, there exists $z \in A$ such that

$$
\sum_{i=1}^{n} \beta_{i}(y) g\left(x_{i}, y\right) \leqslant \sum_{i=1}^{n} \beta_{i}(y) g\left(x_{i}, z\right)
$$

(iii) $g$ is concave on $X$.

Then

$$
\min _{y \in Y} \sup _{x \in X} g(x, y)=\sup _{x \in X} \inf _{y \in Y} g(x, y) .
$$

Theorem 11 (and hence Theorem 10) generalises a well-known minimax theorem of Kneser [21].

Note that $\min _{y \in Y} \sup _{x \in X} g(x, y)$ is attained at some point in $Y$ since $y \rightarrow \sup _{x \in X} g(x, y)$ is also lower semicontinuous and $Y$ is compact.

REMARK. The condition (ii) of Theorem 10 (respectively Theorem 11) can be replaced by the following condition (ii)' without affecting the conclusion:

(ii)' for any finite subset $\left\{x_{1}, \ldots, x_{n}\right\}$ of $X$ and for any $\alpha_{1}, \ldots, \alpha_{n} \geqslant 0$ with $\sum_{i=1}^{n} \alpha_{i}=1$, the following property holds: for any $\lambda \in R$ and for any $A \in \mathcal{F}(Y)$, if $A \subset\left\{y \in Y: \sum_{i=1}^{n} \alpha_{i} g\left(x_{i}, y\right) \leqslant \lambda\right\}$, then

$$
F_{A} \subset\left\{y \in Y: \sum_{i=1}^{n} \alpha_{i} g\left(x_{i}, y\right) \leqslant \lambda\right\}
$$


Proof: It suffices to show that (ii)' $\Rightarrow$ (ii). If (ii) were not true, then there exist $\left\{x_{1}, \ldots, x_{n}\right\} \subset X, \beta_{1}, \ldots, \beta_{n}: Y \rightarrow[0,1]$ with $\sum_{i=1}^{n} \beta_{i}(y)=1$ for each $y \in Y, A \in \mathcal{F}(Y)$ and $\hat{y} \in F_{A}$ such that

$$
\sum_{i=1}^{n} \beta_{i}(\widehat{y}) g\left(x_{i}, \widehat{y}\right)>\sum_{i=1}^{n} \beta_{i}(\widehat{y}) g\left(x_{i}, z\right) \text { for all } z \in A .
$$

Let $\lambda=\max _{z \in A} \sum_{i=1}^{n} \beta_{i}(\widehat{y}) g\left(x_{i}, z\right)$, then

$$
A \subset\left\{y \in Y: \sum_{i=1}^{n} \beta_{i}(\widehat{y}) g\left(x_{i}, y\right) \leqslant \lambda\right\}
$$

By (ii)', $F_{A} \subset\left\{y \in Y: \sum_{i=1}^{n} \beta_{i}(\widehat{y}) g\left(x_{i}, y\right) \leqslant \lambda\right\} ;$ as $\widehat{y} \in F_{A}$, we must have $\sum_{i=1}^{n} \beta_{i}(\widehat{y}) g\left(x_{i}, \widehat{y}\right) \leqslant \lambda$ which contradicts $\left(^{*}\right)$.

Similarly, the condition (iii) of Theorem 8 (respectively, Theorem 9) can be replaced by the following condition (iii)' without affecting the conclusion:

(iii)' for each finite subset $\left\{g_{1}, \ldots, g_{n}\right\}$ of $G$ and for any $\alpha_{1}, \ldots, a_{n} \geqslant 0$ with $\sum_{i=1}^{n} \alpha_{i}=1$, the following property holds: for any $\lambda \in R$ and for any $A \in \mathcal{F}(X)$, if $A \subset\left\{x \in X: \sum_{i=1}^{n} \alpha_{i} g_{i}(y) \leqslant \lambda\right\}$, then

$$
F_{A} \subset\left\{x \in X: \sum_{i=1}^{n} \alpha_{i} g_{i}(x) \leqslant \lambda\right\} .
$$

\section{REFERENCES}

[1] G. Allen, 'Variational inequalities, complementarity problems and duality theorems', $J$. Math. Anal. Appl. 58 (1977), 1-10.

[2] J.S. Bae, W.K. Kim and K.-K. Tan, 'Another generalization of Ky Fan's minimax inequality and its application', (submitted).

[3] C. Bardaro and R. Ceppitelli, 'Some further generalizations of Knaster- KuratowskiMazurkiewicz theorem and minimax inequalities', J. Math. Anal. Appl. 132 (1988), 484-490.

[4] H. Brezis, L. Nirenberg and G. Stampacchia, 'A remark on Ky Fan's minimax principle', Boll. Un. Mat. Ital. 6 (1972), 293-300. 
[5] F.E. Browder, 'The fixed point theory of multi-valued mappings in topological vector spaces', Math. Ann. 177 (1988), 283-301.

[6] F.E. Browder, 'Coincidence theorems, minimax theorems and variational inequalities', in Conference in Modern Analysis and Probability (New Haven, Conn., 1982): Contemp. Math. 26, pp. 67-80 (Amer. Math. Soc., Providence, R.I., 1984).

[7] X.P. Ding and K.-K. Tan, $A$ minimax inequality with applications to existence of equilibrium point and fixed point theorems, Presented to the International Conference on Fixed Point Theory and Its Applications (Marseille-Luminy, France, June 5 - 9, 1989).

[8] X.P. Ding and K.-K. Tan, 'Fixed point theorems and minimax inequalities without convexity', (submitted).

[9] X.P. Ding and K.-K. Tan, 'Matching theorems, fixed point theorems and minimax inequalities without convexity', J. Austral. Math. Soc (to appear).

[10] X.P. Ding and K.-K. Tan, 'Fixed point theorems and equilibria of non-compact generalized games', (submitted).

[11] K. Fan, 'Minimax theorems', Proc. Nat. Acad. Sci. U.S.A. 39 (1953), 42-47.

[12] K. Fan, 'Existence theorems and extreme solutions for inequalities concerning convex functions or linear transformations', Math. Z. 68 (1957), 205-216.

[13] K. Fan, 'A generalization of Tychonoff's fixed point theorem', Math. Ann. 142 (1961), 305-310.

[14] K. Fan, 'A minimax inequality and applications', in Inequalities III, Editor O. Shisha, pp. 103-113 (Academic Press, New York, 1972).

[15] K. Fan, 'Some properties of convex sets related to fixed point theorems', Math. Ann. 266 (1984), 519-537.

[16] A. Granas and F.C. Liu, 'Remark on a theorem of $\mathrm{Ky}$ Fan concerning systems of inequalities', Bull. Inst. Math. Acad. Sinica 11 (1983), 639-643.

[17] C. Horvath, 'Points fixes et coincidences pour les applications multivoques sans convexité', C. R. Acad. Sci. Paris 296 (1983), 403-406.

[18] C. Horvath, 'Points fixes et coincidences dans les espaces topologiques compacts contractiles', C. R. Acad. Sci. Paris 299 (1984), 519-521.

[19] C. Horvath, 'Some results on multivalued mappings and inequalities without convexity', in Nonlinear and Convex Analysis: Lecture Notes in Pure and Appl. Math. 107, Editors B.L. Lin and S. Simons, pp. 99-106 (Dekker, New York, 1987).

[20] B. Knaster, C. Kuratowski and S. Mazurkiewicz, 'Ein Beweis des Fixpunksatzes für n-dimensionale Simplexe', Fund. Math. 14 (1929), 132-137.

[21] H. Kneser, 'Sur un theórèmé fondamental de la théorie des jeux', C. R. Acad. Sci. Paris 234 (1952), 2418-2420.

[22] G. Mehta and E. Tarafdar, 'Infinite dimensional Gale-Nikaido-Debreu theorem and a fixed-point theorem of Tarsfdar', J. Economy Theory (1987), 333-339.

[23] A. Pietsch, Operator Ideals (North-Holland, Amsterdam, 1980).

[24] M.H. Shih and K.-K. Tan, 'The Ky Fan minimax principle, sets with convex sections and variational inequalities', in Differential Geometry, Calculus of Variations and Their Applications: Lecture Notes in Pure and Appl. Math. 100, Editors M. Rassias and T. 
Rassias, pp. 471-481 (Dekker, New York, 1985).

[25] M.H. Shih and K.-K. Tan, 'A geometric property of convex sets with applications to minimax type inequalities and fixed point theorems', J. Austral. Math. Soc. 45 (Ser. A) (1988), 169-183.

[26] M.H. Shih and K.-K. Tan, 'A minimax inequality and Browder-HartmanStampacchia variational inequalities for multi-valued monotone operators' (Proceedings of the Fourth Franco-SEAMS Conference, Chiang Mai, Thailand, 1988).

[27] K.-K. Tan, 'Comparison theorems on minimax inequalities, variational inequalities and fixed point theorems', J. London Math. Soc. 23 (1983), 555-562.

[28] E. Tarafdar, 'On nonlinear variational inequalities', Proc. Amer. Math. Soc. 67 (1977), 95-98.

[29] E. Tarafdar and H.B. Thompson, 'On Fan's minimax principle', J. Austral. Math. Soc. 26 (Ser. A) (1978), 220-226.

[30] C.L. Yen, 'A minimax inequality and its applications to variational inequalities', Pacific J. Math. 97 (1981), 277-481.

[31] J.X. Zhou and G. Chen, 'Diagonal convexity conditions for problems in convex analysis and quasi-variational inequalities', J. Math. Anal. Appl. 132 (1988), 213-225.

Department of Mathematics

Sichuan Normal University

Chengdu, Sichuan

China

Department of Mathematics

Statistics and Computing Science

Dalhousie University

Halifax, Nova Scotis

Canada B3H $3 J 5$
Department of Mathematics Education Chungbuk National University Cheongju

Korea 